\title{
The Shifting Landscape in Utilization of Inpatient, Observation, and Emergency Department Services Across Payers
}

\author{
Teryl K. Nuckols, MD, MSHS ${ }^{1,2 *}$, Kathryn R. Fingar, PhD, MPH${ }^{3}$, Marguerite Barrett, $\mathrm{MS}^{4}$, Claudia A. Steiner, MD, MPH${ }^{5}$, \\ Carol Stocks, PhD, RN , Pamela L. Owens, $\mathrm{PhD}^{6}$
}

\begin{abstract}
${ }^{1}$ Rand Corporation, Santa Monica, California; 2Division of General Internal Medicine, Department of Medicine, Cedars-Sinai, Los Angeles, California; ${ }^{3}$ Truven Health Analytics, Ann Arbor, Michigan; ${ }^{4}$ M.L. Barrett, Inc., Del Mar, California; ${ }^{5}$ Affiliation during this investigation: Agency for Healthcare Research and Quality, Rockville, Maryland; current affiliation: Kaiser Permanente Colorado, Denver, Colorado; ${ }^{6}$ Agency for Healthcare Research and Quality, Rockville, Maryland.
\end{abstract}

Recent policies by public and private payers have increased incentives to reduce hospital admissions. Using data from four states from the Agency for Healthcare Research and Quality Healthcare Cost and Utilization Project, this study compared the payer-specific population-based rates of adults using inpatient, observation, and emergency department (ED) services for 10 common medical conditions in 2009 and in 2013. Patients had an expected primary payer of private insurance, Medicare, Medicaid, or no insurance. Across all four payer populations, inpatient admissions declined, and care shifted toward treat-and-release observation stays and ED visits. The percentage of hospitalizations that began with an observation stay increased. Implications for quality of care and costs to patients warrant further examination. Journal of Hospital Medicine 2017;12:443-446. () 2017 Society of Hospital Medicine
For over a decade, private and public payers have implemented policies aimed at reducing rates of inpatient hospitalization. One approach for doing so is to improve ambulatory care, which can reduce the need for hospital-based acute care. Another approach is to stabilize acutely ill patients and discharge them from the emergency department (ED) or following a period of observation. ${ }^{1}$ Private payers are entering into value-based contracting arrangements with hospitals and health systems to improve the quality of ambulatory care and lower healthcare expenditures. ${ }^{2}$ Enrollment in managed care programs has grown among Medicaid recipients for similar reasons. ${ }^{3}$ Policies of the Centers for Medicare \& Medicaid Services (CMS) encourage improvements in ambulatory care as well as observation of Medicare beneficiaries instead of inpatient admission in certain situations. ${ }^{4}$

Recent studies have documented declines in inpatient admissions and increases in treat-and-release observation stays and ED visits among Medicare beneficiaries. ${ }^{4-7}$ However, almost half of all hospitalizations unrelated to childbirth occur among patients with private insurance, Medicaid, or no insurance. ${ }^{8}$ Less is known about shifts in the nature of hospital-based acute care among these populations. Such shifts

\footnotetext{
*Address for correspondence and reprint requests: Teryl K. Nuckols, MD, MSHS, Health Services Researcher, RAND Corporation, 1776 Main Street, Santa Monica, CA 90401; Associate Professor and Director, Division of General Internal Medicine, Cedars-Sinai Medical Center, 8700 Beverly Boulevard, Los Angeles, CA 90048; Telephone: 310-423-2760; Fax: 310-423-0436; E-mail: teryl.nuckols@cshs.org

Additional Supporting Information may be found in the online version of this article.

Received: August 26, 2016; Revised: December 1, 2016; Accepted: December 2, 2016
}

2017 Society of Hospital Medicine DOI 10.12788/jhm.2751 would have implications for quality of care, patient outcomes, and costs. Therefore, further investigation is warranted.

Our objective was to investigate recent trends in payerspecific population-based rates of adults using inpatient, observation, and ED services. We focused on 10 medical conditions that are common reasons for hospital-based acute care: heart failure, bacterial pneumonia, chronic obstructive pulmonary disease, asthma, dehydration, urinary tract infection, uncontrolled diabetes, diabetes with long-term complications, diabetes with short-term complications, and hypertension. These conditions constitute more than $20 \%$ of inpatient stays in the general medical service line, can be affected by improvements in ambulatory care, and provided a consistent set of diagnoses to track trends over time. ${ }^{9}$ We used 2009 and 2013 data from four states to examine trends among individuals with private insurance, Medicare, Medicaid, and no insurance.

\section{METHODS}

We obtained encounter-level data for Georgia, Nebraska, South Carolina, and Tennessee from the Agency for Healthcare Research and Quality (AHRQ), Healthcare Cost and Utilization Project (HCUP). ${ }^{10}$ Using encrypted patient identifiers, we linked inpatient admissions from the 2009 and 2013 State Inpatient Databases, observation stays from the State Ambulatory Surgery and Services Databases, and ED visits from State Emergency Department Databases.

We defined the 10 medical conditions using numerator specifications from the ICD-9-CM v 5.0 AHRQ Prevention Quality Indicators (see Appendix). At most, 1 inpatient admission, 1 observation stay, and $1 \mathrm{ED}$ visit for a study condition was counted for each adult in each year. Limiting the number of visits minimized the skew caused by multiple uses of the same service. 


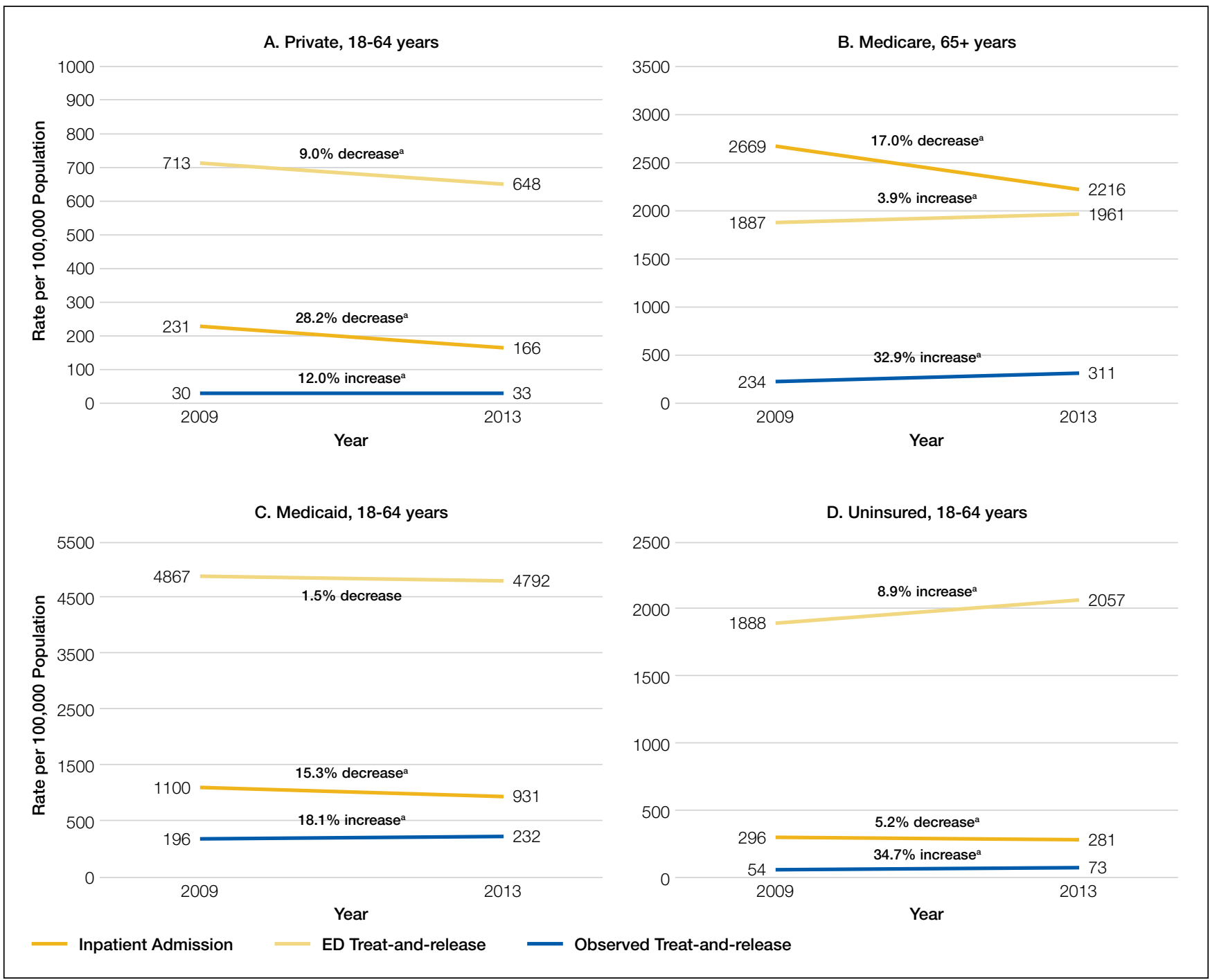

FIG. 1. Trends in the rate of adults (per 100,000 population) with treat-and-release observation stays and ED visits relative to inpatient admissions for ambulatory care sensitive conditions, 2009-2013.

a $<<0$.05. Sources: Agency for Healthcare Research and Quality, Healthcare Cost and Utilization Project, state databases and U.S. Census Bureau, American Community Survey, Georgia, Nebraska, South Carolina, and Tennessee, 2009 and 2013.

NOTE: Abbreviation: ED, emergency department.

Using the American Community Survey, we calculated utilization rates for each type of service per 100,000 population in four payer and age groups: privately insured adults, Medicaid recipients, and uninsured adults 18 to 64 years, as well as Medicare beneficiaries 65 years and older. For each group, we also examined the origin of inpatient admissionsthose who were directly admitted without evaluation in the $\mathrm{ED}$, those admitted from the ED, and ED visits leading to observation stays and then inpatient admission.

\section{RESULTS}

Comparing 2009 and 2013, population-based rates of adults with 1 or more inpatient admissions for 10 common medical conditions declined, whereas rates of adults with treat-andrelease observation stays rose. Changes in rates of treat-andrelease ED visits varied across payers but were small relative to the substantial declines in inpatient admissions (Figure 1). In addition, a growing percentage of inpatient admissions began as observation stays and fewer adults were admitted directly, except among uninsured individuals (Figure 2).

\section{Private Payers, 18 to 64 Years}

The rate of adults with treat-and-release observation stays rose $(+12.0 \%, 30$ to 33 per 100,000 private payer population, $P<0.001)$. The rate of adults with treat-and-release ED visits declined $(-9.0 \%, 713$ to 648 per 100,000 population, $P<0.001$ ), but by less than for inpatient admissions (-28.2\%, 231 to 166 per 100,000 population, $P<0.001$; Figure $1 \mathrm{~A})$. The percentage of inpatient admissions that began as observation stays rose (from $4.1 \%$ to $5.4 \%, P=0.041$ ), as did the percentage of admissions originating in the ED (from $66.4 \%$ to $71.5 \%, P \leq 0.001$; Figure 2 ). 


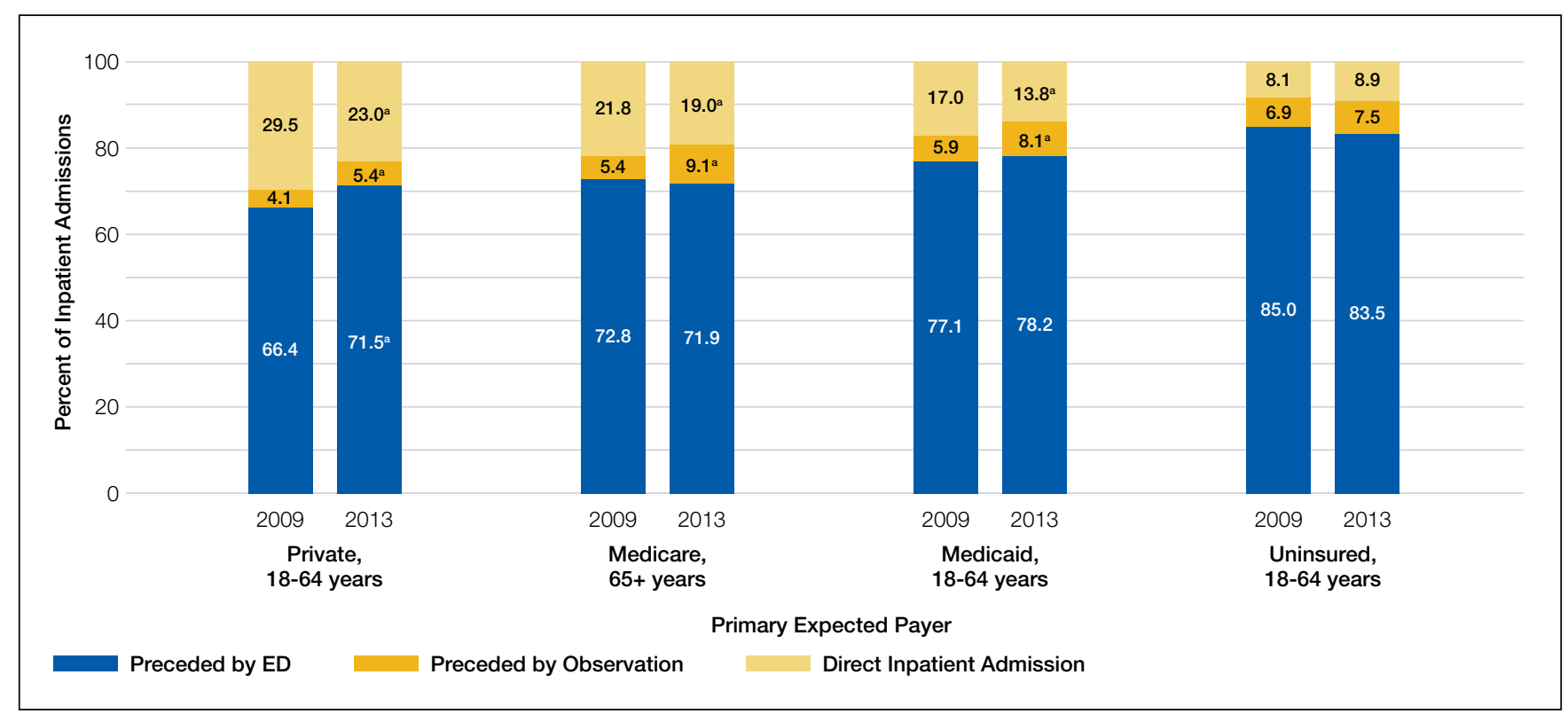

FIG. 2. Trends in the proportion of inpatient admissions for ambulatory care sensitive conditions that were preceded by observation or ED care. a $P<0.05$. Sources: Agency for Healthcare Research and Quality, Healthcare Cost and Utilization Project, State Inpatient Databases, Georgia, Nebraska, South Carolina, and Tennessee, 2009 and 2013. NOTE: Abbreviation: ED, emergency department.

\section{Medicare, 65 Years and Older}

The rate of adults with inpatient admissions declined (-17.0\%, 2669 to 2216 per 100,000 Medicare population, $P$ $<0.001)$. Rates rose for adults with treat-and-release ED visits ( $+3.9 \%, 1887$ to 1961 per 100,000 population, $P<0.001)$ and treat-and-release observation stays ( $+32.9 \%, 234$ to 311 per 100,000 population, $P<0.001$; Figure $1 B)$. The percentage of inpatient admissions that began as observation stays also rose $(5.4 \%$ to $9.1 \%, P<0.001$; Figure 2$)$.

\section{Medicaid, 18 to 64 Years}

The rate of adults with inpatient admissions declined (-15.3\%, 1100 to 931 per 100,000 Medicaid population, $P<0.001)$, whereas treat-and-release ED visits remained flat $(-1.5 \%, 4867$ to 4792 per 100,000 population, $P=0.413)$ and treat-and-release observation stays rose $(+18.1 \%, 196$ to 232 per 100,000 population, $P<0.001$; Figure $1 C)$. The percentage of inpatient admissions that began as observation stays rose $(5.9 \%$ to $8.1 \%, P=0.022$; Figure 2$)$.

\section{Uninsured, 18 to 64 Years}

The rate of adults with inpatient admissions declined $(-5.2 \%, 296$ to 281 per 100,000 uninsured population, $P$ $=0.003)$, whereas rates rose for treat-and-release ED visits (+8.9\%, 1888 to 2057 per 100,000 population, $P<0.001)$ and treat-and-release observation stays $(34.7 \%, 54$ to 73 per 100,000 population, $P<0.001$; Figure 1D). The source of inpatient admissions remained stable (Figure 2 ).

\section{DISCUSSION}

Data on hospital encounters from four states show that both ED visits and observation stays are playing an increasing role in hospital-based acute care for 10 common conditions among populations insured by private payers, Medicare, and Medicaid, as well as those without insurance. Compared with 2009, in 2013 substantially fewer individuals had inpatient admissions, and patients were more likely to be discharged from the ED or discharged following observation without receiving inpatient care. Additionally, an increasing percentage of inpatient admissions followed observation stays, whereas direct admissions declined.

Previous authors also have reported declines in inpatient stays for these same conditions. ${ }^{11}$ Others have reported increases in the use of observation stays for diverse conditions among patients with private insurance, Medicare beneficiaries, and veterans. ${ }^{4,12,13}$ The unique attributes of HCUP databases from these four states (eg, all-payer data including patient linkage numbers across inpatient, observation, and ED care) enabled us to assess concurrent shifts in hospital-based acute care from inpatient to outpatient care among multiple payer populations. A recent analysis reported declines in readmissions and increases in observation visits occurring within 30 days after hospitalization among Medicare beneficiaries with heart failure, acute myocardial infarction, or pneumonia. ${ }^{14}$ Future research should examine trends in readmissions and observation visits following hospitalization among multiple payer populations.

These shifts raise two important questions. The first pertains to quality of care, including outcomes. Although dedicated observation units with condition-specific care pathways can be associated with shorter stays and fewer admissions, many patients placed under observation are neither in dedicated units nor subject to care pathways. ${ }^{15,16}$ Systems for monitoring quality of care are less developed for 
observation care. The CMS publicly reports hospital-level data on quality of ED and inpatient care, including for several of the conditions we studied, but no measures apply to observation stays. ${ }^{17}$ Little is known about whether shifts from inpatient care to observation status or discharge from the ED are associated with different health outcomes.

The second issue is patients' out-of-pocket costs. Although shifts from inpatient admissions to observation stays can reduce costs to payers, ${ }^{15}$ effects on patient out-of-pocket costs are uncertain and may vary. For privately insured patients, out-of-pocket costs may be up to four times higher for observation than for inpatient care..$^{18}$ For Medicare beneficiaries, out-of-pocket costs can be higher for observation than for inpatient stays, particularly when patients receive costly medications or are discharged to skilled nursing facilities; ${ }^{19,20}$ however, having secondary insurance dramatically reduces out-of-pocket costs. ${ }^{21}$ We are not aware of data on Medicaid recipients or uninsured individuals.

This study has limitations. Only four states had data needed for these analyses, so generalization to other states is limited. Our analysis was descriptive and did not control for case mix, evaluate specific policies by any payer, or assess the full volume of visits among high utilizers. Movement of healthier or sicker individuals across payers could have contributed to temporal trends, but findings were similar across payers.

In conclusion, among 10 common medical conditions and three major payer populations and uninsured individuals in four states, inpatient admissions declined, and care shifted toward treat-and-release ED visits and observation stays. The number of inpatient admissions that began as observation stays also increased. Given these trends and the possibility that such shifts may be widespread and continue beyond 2013, quality of care, outcomes, and costs to patients warrant further evaluation.

\section{Acknowledgments}

The authors gratefully acknowledge Minya Sheng, MS (Truven Health Analytics) for assistance in programming and data management, and Paige Jackson, MS and Linda Lee, PhD, (Truven Health Analytics) for providing editorial review of the manuscript. They also wish to acknowledge the four HCUP Partner organizations that contributed to the 2009 and 2013 HCUP state databases used in this study: Georgia Hospital Association, Nebraska Hospital Association, South Carolina Revenue and Fiscal Affairs Office, and Tennessee Hospital Association.

Disclosure: Funding for this study was provided by the Agency for Healthcare Research and Quality (AHRQ), Healthcare Cost and Utilization Project (HCUP) (Contract No. HHSA-290-2013-00002-C). The views expressed in this article are those of the authors and do not necessarily reflect those of the Agency for Healthcare Research and Quality or the U.S. Department of Health and Human Services. The authors have no conflicts of interest to declare or financial disclosures.

\section{References}

1. Ross MA, Hockenberry JM, Mutter R, Barrett M, Wheatley M, Pitts SR. Protocol-driven emergency department observation units offer savings, shorter stays, and reduced admissions. Health Aff (Millwood). 2013;32(12):2149-2156
2. Song Z. Accountable care organizations in the U.S. health care system. J Clin Outcomes Manag. 2014;21(8):364-371.

3. Kaiser Family Foundation. Total Medicaid MCOs. State Health Facts. 2016. http://kff.org/other/state-indicator/total-medicaid-mcos/. Accessed July 19, 2016.

4. Feng Z, Wright B, Mor V. Sharp rise in Medicare enrollees being held in hospitals for observation raises concerns about causes and consequences. Health Aff (Millwood). 2012;31(6):1251-1259.

5. Skinner HG, Blanchard J, Elixhauser A. Trends in emergency department visits, 2006-2011. HCUP Statistical Brief \#179. September 2014. Agency for Healthcare Research and Quality, Rockville, MD. http://www.hcup-us.ahrq. gov/reports/statbriefs/sb179-Emergency-Department-Trends.pdf. Accessed July 21, 2016.

6. Medicare Payment Advisory Commission. Report to the Congress: Medicare and the Health Care Delivery System. June 2015. http://www.medpac.gov/docs/ default-source/reports/june-2015-report-to-the-congress-medicare-and-thehealth-care-delivery-system.pdf?sfvrsn=0. Accessed October 6, 2016.

7. Medicare Payment Advisory Commission. Report to the Congress: Medicare Payment Policy. March 2016. http://www.medpac.gov/docs/default-source/ reports/march-2016-report-to-the-congress-medicare-payment-policy.pdf?sfvrsn=0. Accessed October 6, 2016.

8. Agency for Healthcare Research and Quality. Healthcare Cost and Utilization Project. HCUPnet. Agency for Healthcare Research and Quality, Rockville, MD. http://hcupnet.ahrq.gov/. Accessed October 6, 2016.

9. Fingar KR, Barrett ML, Elixhauser A, Stocks C, Steiner CA. Trends in potentially preventable inpatient hospital admissions and emergency department visits. HCUP Statistical Brief \#195. November 2015. Agency for Healthcare Research and Quality, Rockville, MD. http://www.hcup-us.ahrq.gov/reports/ statbriefs/sb195-Potentially-Preventable-Hospitalizations.pdf. Accessed August 9, 2016.

10. Agency for Healthcare Research and Quality. HCUP Databases. Agency for Healthcare Research and Quality, Rockville, MD. www.hcup-us.ahrq.gov/databases.jsp. Accessed August 8, 2016.

11. Torio CM, Andrews RM. Geographic variation in potentially preventable hospitalizations for acute and chronic conditions, 2005-2011. HCUP Statistical Brief, \#178. September 2014. Agency for Healthcare Research and Quality, Rockville, MD. http://www.hcup-us.ahrq.gov/reports/statbriefs/sb178-Preventable-Hospitalizations-by-Region.pdf. Accessed November 8, 2015.

12. Wright B, O'Shea AM, Ayyagari P, Ugwi PG, Kaboli P, Vaughan Sarrazin M. Observation rates at veterans' hospitals more than doubled during 2005-13, similar to Medicare trends. Health Aff (Millwood). 2015;34(10):1730-1737.

13. Noel-Miller C, Lind K. Is observation status substituting for hospital readmission? Health Affairs Blog. October 28, 2015. Project Hope: The People-to-People Health Foundation, Inc., Millwood, VA. http://healthaffairs.org/ blog/2015/10/28/is-observation-status-substituting-for-hospital-readmission/. Accessed November 8, 2015.

14. Zuckerman RB, Sheingold SH, Orav EJ, Ruhter J, Epstein AM. Readmissions, observation, and the hospital readmissions reduction program. $N$ Engl J Med. 2016;374(16):1543-1551

15. Ross MA, Hockenberry JM, Mutter R, Barrett M, Wheatley M, Pitts SR. Protocol-driven emergency department observation units offer savings, shorter stays, and reduced admissions. Health Aff (Millwood). 2013;32(12):2149-2156.

16. Sheehy AM. Dedicated observation unit for patients with "observation status" -- reply. JAMA Intern Med. 2014;174(2):301-302.

17. Medicare.gov. Measures and current data collection periods. Centers for Medicare and Medicaid Services, Baltimore, MD. https://www.medicare.gov/hospitalcompare/Data/Data-Updated.html\#. Accessed July 19, 2016.

18. Jaffe S. You're being observed in the hospital? Patients with private insurance better off than seniors. September 11, 2014. Kaiser Health News, Kaiser Family Foundation, Menlo Park, CA. http://khn.org/news/youre-being-observed-inthe-hospital-patients-with-private-insurance-are-better-off-than-seniors/. Accessed November 8, 2015.

19. Kangovi S, Cafardi SG, Smith RA, Kulkarni R, Grande D. Patient financial responsibility for observation care. J Hosp Med. 2015;10(11):718-723.

20. U.S. Department of Health and Human Services, Office of Inspector General. Hospitals' use of observation stays and short inpatient stays for Medicare beneficiaries. Memorandum Report OEI-02-12-00040. July 29, 2013. U.S. Department of Health and Human Services, Washington, DC. https://oig.hhs.gov/oei/ reports/oei-02-12-00040.pdf. Accessed October 6, 2016.

21. Doyle BJ, Ettner SL, Nuckols TK. Supplemental insurance reduces out-of-pocket costs in Medicare observation services. J Hosp Med. 2016;11(7):502-504. 